BI-TP 94/27

May 1994

\title{
Effective Scalar Field Theory for the Electroweak Phase Transition
}

\author{
F. Karsch ${ }^{1}$, T. Neuhaus ${ }^{1}$ and A. Patkós ${ }^{2}$
}

\begin{abstract}
:
We investigate an effective model for the finite temperature symmetry restoration phase transition of the electroweak theory. It is obtained by dimensional reduction of the $3+1$ dimensional full theory and by subsequent integration over all static gauge degrees of freedom. The resulting theory corresponds to a 3-dimensional $O(4)$ ferromagnet containing cubic and quartic terms of the field in its potential function. Possible nonperturbative effects of a magnetic screening mass are parametrically included in the potential. We analyse the theory using mean field and numerical Monte Carlo (MC) simulation methods. At the value of the physical Higgs mass, $m_{H}=37 \mathrm{GeV}$, considered in the present investigation, we find a discontinuous symmetry restoring phase transition. We determine the critical temperature, order parameter jump, interface tension and latent heat characteristics of the transition. The Monte Carlo results indicate a somewhat weaker first order phase transition as compared to the mean field treatment, demonstrating that nonperturbative fluctuations of the Higgs field are relevant. This effect is especially important for the interface tension. Any observation of hard first order transition could result only from non-perturbative effects related to the gauge degrees of freedom.
\end{abstract}

\footnotetext{
${ }^{1}$ Fakultät für Physik, Universität Bielefeld, D-33615 Bielefeld, FRG

${ }^{2}$ Department of Atomic Physics, Eötvös University, H-1088, Puskin u. 5-7, Budapest, Hungary
} 


\section{Introduction}

The tiny matter-antimatter asymmetry observed on cosmic scales requires explanation in the framework of standard cosmology. Baryon number violating processes are known to occur in the electro-weak theory and are, in fact, expected to occur frequently at high temperatures in equilibrium. The occurence of a non-vanishing matter-antimatter asymmetry, however, is expected to be possible only, if the universe evolved through some nonequilibrium stages. If the electroweak phase transition was of first order (discontinuous) nature, then at temperatures $T_{c} \sim 100 \mathrm{GeV}$ there might have been a chance for developing the observed baryon asymmetry of the universe. The very active present day investigation of this question is based predominantly on a perturbative evaluation of the effective potential of the Higgs-field.

The interest in non-perturbative studies stems from two sources - the generation of a magnetic mass as well as the breakdown of the perturbative treatment in the case of a weakly first order phase transition. A nonperturbatively generated screening mass of the magnetic part of the gauge field fluctuations is expected to weaken the discontinuous nature of the transition. Eventually this could lead to a second order transition, if the magnetic screening mass becomes too large [1]. Thus the perturbative calculations [2, 3] lead to constraints on the maximal magnetic screening mass allowed for a first order transition and also lead to other predictions, like for instance the latent heat and surface tension, important for discussing the kinetics of the phase transformation.

The validity of any perturbative treatment of the transition has been questioned for first order transitions where near $T_{c}$ the ratio $T / m\left(\Phi_{\min }\right)$ be-

comes large (weakly first order transition). In this case the fluctuations of the light modes (especially of the Goldstone modes) might require special considerations. Very recently various exploratory studies have been performed, in which it has been attempted to apply renormalization group theory of critical phenomena to this situation [四, 5, 6]. However, one has to emphasize that techniques like the $\epsilon$-expansion were successful in finding universal characterisations, mainly critical exponents. Their success for calculating critical temperatures, order parameter discontinuities or surface tensions is by no means guaranteed.

Under these circumstances numerical simulations seem to be an especially 
valuable source of information. Exploratory investigations of the full, 4-d finite temperature theory met difficulties because of the large fluctuations due to the weakly coupled nature of the phenomena [7]. In this situation dimensional reduction might prove to be of important practical help [8, 9].

In this work we study the influence of the finite temperature fluctuations of the Higgs-field on these parameters. In particular we will examine an effective 3-d theory for the Higgs-field, which has been obtained from the $(3+1)$-dimensional SU(2)-Higgs model in two steps:

i) Dimensional reduction: One integrates over all non-static Matsubara fields at one-loop level. Since these modes are massive, no infrared sensitivity is expected, their perturbative integration seems to be wellfounded.

ii) Elimination of gauge degrees of freedom: The theory resulting from the first step is a 3-dimensional Gauge-Higgs model, where in addition, also an isovector field, the fourth component of the gauge fields, is present. In order to reduce the theory further one integrates over the magnetic gauge degrees of freedom and the isovector scalar. According to the improved perturbative treatments, the infrared stability can be ensured, if one includes into the result of the "naive" 3-dimensional 1loop calculation a magnetic and an electric screening mass. Especially the first of them lacks, however, firm theoretical basis.

We remark that the resulting effective scalar theory has been used recently also for the investigation of bubble nucleation in the electroweak phase transition [10].

In section 2 we first present the result of step i) for an $\mathrm{SU}(2)$ Higgs-model with $\mathrm{N}$ doublets:

$$
S=\int_{0}^{\beta} d \tau \int d^{3} x\left[\frac{1}{4} F_{\mu \nu}^{a} F_{\mu \nu}^{a}+\frac{1}{2}\left(D_{\mu} \Phi_{q}\right)^{+}\left(D_{\mu} \Phi_{q}\right)+\frac{1}{2} m^{2} \Phi_{q}^{+} \Phi_{q}+\frac{\lambda}{24}\left(\Phi_{q}^{+} \Phi_{q}\right)^{2}\right]+\text { c.t. },
$$

$\left(q=1, \ldots, N ; \mu=1, . ., 4 ; a=1,2,3 ; D_{\mu} \Phi=\left(\partial_{\mu}+i g A_{\mu}^{a} \tau^{a} / 2\right) \Phi\right)$.

In the limiting case $\mathrm{N}=\infty$ step ii) can be performed exactly and a pure scalar action of very similar form to that actually studied in later sections is found. Also, some evidence will be presented from the perturbative analysis of the 3-d effective high temperature action of the $N=1$ Higgs model that in 
the coupling region corresponding to small Higgs mass values the pure scalar model might offer considerable insight into the physics of the electroweak phase transition.

The discretisation of the effective model is described in section 3 . The continuum limit of its mean field solution will be discussed in order to clarify the strategy of the non-perturbative investigations. Special attention will be paid to the uncertainties in taking the continuum limit, arising from the application of different renormalisation conditions.

Section 4 presents a detailed discussion of the Monte Carlo simulation of the effective scalar model. Several physical quantities relevant to the phase transition will be stochastically evaluated and compared carefully to the information available from the literature. In the simulation the mass of the Higgs field was chosen approximately $m_{H}(T=0) \sim 35 \mathrm{GeV}$. Conclusions will be drawn in section 5 .

\section{Derivation of the Effective Scalar Action}

In this section we outline the derivation of the effective 3-d action for the SU(2) Higgs model with N scalar doublets on the 1-loop level. It represents a natural framework for the investigation of the finite temperature electroweak phase transition in an appropriate large $N$ limit [6, 11]. It turns out that the resulting 3-d action can be shown to be strictly equivalent to an effective pure scalar model. In this sense the model provides motivating background for the investigation of the scalar effective model in the case of a single scalar doublet $(\mathrm{N}=1)$.

\subsection{Effective Action in the Large N Limit}

Choosing the nonvanishing Higgs-field vacuum expectation value, $\Phi_{0}$, in the first scalar doublet, the computation naturally breaks up into a part identical with the calculation of the reduced action for the model with one doublet and the other part which consists of calculating the contribution from the appropriately separated group of $\mathrm{N}-1$ doublets. This statement is evident if one considers the quadratic part of the scalar action alone. The Higgs 
doublets are parametrised as:

$$
\Phi_{q}=\left(\begin{array}{c}
\Phi_{q 1} \\
\Phi_{q 2}
\end{array}\right)=\delta_{q, 1}\left(\begin{array}{c}
0 \\
\Phi_{0}
\end{array}\right)+\left(\begin{array}{c}
\xi_{q 1}+i \xi_{q 2} \\
\xi_{q 3}+i \xi_{q 4}
\end{array}\right), \quad q=1, \ldots, N
$$

Their potential is $\mathrm{O}(4 \mathrm{~N})$-symmetric and depends only on the length of the $4 \mathrm{~N}$-component Higgs-field vector:

$$
U_{\text {Higgs }}=\frac{1}{2} m^{2} \Phi_{q}^{+} \Phi_{q}+\frac{1}{24} \lambda\left(\Phi_{q}^{+} \Phi_{q}\right)^{2} .
$$

The quadratic action derived with eq.(2) and eq.(3) has the form:

$$
S_{\mathrm{Higgs}}^{(2)}=\frac{1}{2}\left(m^{2}+\frac{\lambda}{6} \Phi_{0}^{2}\right) \sum_{q=2}^{N} \sum_{\alpha=1}^{4} \xi_{q \alpha}^{2}+\frac{1}{2}\left(m^{2}+\frac{\lambda}{6} \Phi_{0}^{2}\right) \sum_{\alpha \neq 3} \xi_{1 \alpha}^{2}+\frac{1}{2}\left(m^{2}+\frac{\lambda}{2} \Phi_{0}^{2}\right) \xi_{13}^{2} .
$$

Clearly, at 1-loop level the fluctuations of the fields with different $q$ are independent, and this is true also for the gauge-scalar coupling part of the action. In the quadratic part of the latter the gauge fields couple only to the $q=1$ doublet, where the static part of the Higgs-configuration was choosen to point to. The fluctuations of the other N-1 doublets coincide with those of $2(\mathrm{~N}-1)$ independent complex scalar fields on a static abelian $A_{0}$ background, which is characterised in the Fourier-space uniformly by the following $2 \times 2$ matrix:

$$
\left(\begin{array}{cc}
K^{2}+m^{2}+\frac{g^{2}}{4} A_{0}^{2}+\frac{\lambda}{6} \Phi_{0}^{2} & i g \omega_{n} A_{0} \\
-i g \omega_{n} A_{0} & K^{2}+m^{2}+\frac{g^{2}}{4} A_{0}^{2}+\frac{\lambda}{6} \Phi_{0}^{2}
\end{array}\right),
$$

$\left(K^{2}=\mathbf{k}^{2}+\omega_{n}^{2}\right)$. The contribution from the coupled gauge-scalar fluctuations has been evaluated before [8, 12. We thus discuss here only the contribution from the additional scalar fields:

$$
\Delta U=(N-1) \sum_{n \neq 0} \int \frac{d^{3} k}{(2 \pi)^{3}} \ln \left[\left(K^{2}+\frac{g^{2}}{4} A_{0}^{2}+m^{2}+\frac{\lambda}{6} \Phi_{0}^{2}\right)^{2}-g^{2} \omega_{n}^{2} A_{0}^{2}\right] .
$$

The usual expansion in the argument of the logarithm leads to modification of the classical action, in which we are going to keep terms up to dim 4 combinations. After performing the frequency sum and the momentum integration 
the following cut-off regularised corrections to the quartic $\Phi_{0}-A_{0}$-potential arise:

$$
\begin{gathered}
\Delta U=(N-1)\left\{\frac{g^{2}}{2} A_{0}^{2}\left(-\frac{\Lambda T}{2 \pi^{2}}+\frac{T^{2}}{6}+\frac{m^{2}}{8 \pi^{2}}\right)+\frac{\lambda}{3} \Phi_{0}^{2}\left(\frac{\Lambda^{2}}{8 \pi^{2}}-\frac{\Lambda T}{2 \pi^{2}}\right.\right. \\
\left.\left.+\frac{T^{2}}{12}+\frac{m^{2}}{8 \pi^{2}}-D_{0} \frac{1}{8 \pi^{2}}\right)+\frac{g^{4}}{192 \pi^{2}} A_{0}^{4}+\frac{g^{2} \lambda}{96 \pi^{2}} A_{0}^{2} \Phi_{0}^{2}+\frac{\lambda^{2}}{36} \Phi_{0}^{4}\left(\frac{1}{8 \pi^{2}}-D_{0} \frac{1}{8 \pi^{2}}\right)\right\}
\end{gathered}
$$

with $D_{0}=\ln \frac{\Lambda}{T}+$ const. Adding eq.(7) to the 1 -doublet+gauge contribution, calculated in the thermal static gauge previously [12], the regularised full potential has the following expression:

$$
\begin{gathered}
U_{\text {static }}\left[A_{0}, \Phi_{0}\right]=\frac{1}{2} m^{2} \Phi_{0}^{2}+\frac{1}{24} \lambda \Phi_{0}^{4} \\
+\frac{1}{2} \Phi_{0}^{2}\left[\left(\frac{9}{4} g^{2}+\frac{2 N+1}{3} \lambda\right)\left(\frac{\Lambda^{2}}{8 \pi^{2}}-\frac{\Lambda T}{2 \pi^{2}}+\frac{T^{2}}{12}-D_{0} \frac{m^{2}}{8 \pi^{2}}\right)+\frac{m^{2}}{8 \pi^{2}}\left(\frac{3 g^{2}}{4}+\frac{(2 N+1) \lambda}{3}\right)\right] \\
+\frac{1}{24} \Phi_{0}^{4}\left(\frac{(N+2) \lambda^{2}}{12 \pi^{2}}+\frac{27 g^{4}}{64 \pi^{2}}+\frac{3 \lambda g^{2}}{16 \pi^{2}}-D_{0}\left(\frac{(N+2) \lambda^{2}}{12 \pi^{2}}+\frac{27 g^{4}}{64 \pi^{2}}+\frac{9 \lambda g^{2}}{16 \pi^{2}}\right)\right) \\
+\frac{N+16}{192 \pi^{2}} g^{4} A_{0}^{4}+\frac{1}{2} A_{0}^{2}\left(\frac{N+4}{6} g^{2} T^{2}+\frac{N g^{2} m^{2}}{8 \pi^{2}}-\frac{N+4}{2 \pi^{2}} g^{2} \Lambda T\right) \\
+\frac{1}{8} g^{2} A_{0}^{2} \Phi_{0}^{2}\left(1+\frac{(2 N+1) \lambda}{24 \pi^{2}}+\frac{3 g^{2}}{16 \pi^{2}}\left(5-3 D_{0}\right)\right) .
\end{gathered}
$$

For the renormalised potential the "classical" extremum conditions are prescribed at the classical position of the minimum [13]:

$$
\frac{d U(T-\text { indep })}{d \Phi_{0}}=0, \quad \frac{d^{2} U(T-\text { indep })}{d \Phi_{0}^{2}}=m_{H}^{2}(T=0), \quad \Phi_{0}=v_{0} .
$$

For the separation of the $T$-independent part one has to rewrite the logarithmic part (proportional to $D_{0}$ ) of the regularised potential conveniently:

$$
\begin{aligned}
-\frac{1}{2}\left[\frac { 1 } { 1 6 \pi ^ { 2 } } m ^ { 2 } \Phi _ { 0 } ^ { 2 } \left(\frac{9 g^{2}}{4}\right.\right. & \left.\left.+\frac{2 N+1}{3} \lambda\right)+\frac{1}{96 \pi^{2}} \Phi_{0}^{4}\left(\frac{(N+2) \lambda^{2}}{3}+\frac{27 g^{4}}{16}+\frac{9 \lambda g^{2}}{4}\right)\right] \ln \frac{\Lambda^{2}}{T^{2}} \\
& =-\frac{1}{64 \pi^{2}} \sum_{Q} n_{Q} m_{Q}^{4}\left(\Phi_{0}\right) \ln \frac{\Lambda^{2}}{T^{2}},
\end{aligned}
$$

$\mathrm{Q}=\mathrm{T}($ ransversal), $\mathrm{L}$ (ongitudinal),H(iggs), $\mathrm{G}$ (oldstone). The most natural choice for $m_{Q}^{2}\left(\Phi_{0}\right)$ is the following:

$$
\begin{aligned}
& n_{T}=6, \quad m_{T}^{2}=\frac{1}{4} g^{2} \Phi_{0}^{2} \\
& n_{H}=1, \quad m_{H}^{2}=m^{2}+\frac{\lambda}{2} \Phi_{0}^{2} \\
& n_{G}=4 N-1, \quad m_{G}^{2}=m^{2}+\frac{\lambda^{\prime}}{6} \Phi_{0}^{2}, \\
& n_{L}=3, \quad m_{L}^{2}=\left(\frac{1}{16}-\frac{27}{16(4 N-1)}\right)^{1 / 2} g^{2} \Phi_{0}^{2}
\end{aligned}
$$


with $\lambda^{\prime}=\lambda+27 g^{2} /(8 N-2)$. For large values of $N m_{L}^{2}$ is real, and the limit $N \rightarrow \infty$ gives

$$
n_{L}=3, \quad m_{L}^{2}=\frac{1}{4} g^{2} \Phi_{0}^{2}=m_{T}^{2}, \quad(N=\infty)
$$

On the right hand side of eq.(10) one can then separate the cut-off dependence from the T-dependence by introducing appropriate normalisation scales, possibly different for each value of $\mathrm{Q}$. For the gauge coupling $g^{2}$ we choose the renormalization scale $\mu=T$ and absorb the logarithmic and finite corrections fully into its counterterm. This is consistent with the omission of the effect of the wave-function renormalisation in the present calculation (c.f. [12]).

After absorbing the T-independent infinities and some finite parts into the counterterms of $m^{2}, \lambda$ and $g^{2}$ one finds the following explicit T-dependent potential fulfilling the conditions eq. (9):

$$
\begin{gathered}
U\left[A_{0}, \Phi_{0}\right]=\frac{1}{2} m^{2} \Phi_{0}^{2}+\frac{1}{24} \lambda \Phi_{0}^{4}+\frac{1}{2} \Phi_{0}^{2}\left(\frac{9 g^{2}}{4}+\frac{(2 N+1) \lambda}{3}\right)\left(\frac{T^{2}}{12}-\frac{\Lambda T}{2 \pi^{2}}\right) \\
-\frac{1}{128 \pi^{2}} m^{2} \Phi_{0}^{2}\left[\frac{\lambda^{\prime}(4 N-1)}{3} \ln \frac{9 g^{2} v_{0}^{2}}{16 T^{2}}+\lambda(4 N-1) \ln \frac{N \lambda v_{0}^{2}}{3 T^{2}}+\frac{27 g^{4}}{\lambda}+\frac{3 g^{2} \lambda^{\prime}}{2 \lambda}+2 \lambda\right] \\
+\frac{1}{1536 \pi^{2}} \Phi_{0}^{4}\left[-\frac{27 g^{4}}{2} \ln \frac{N g^{2} v_{0}^{2}}{4 T^{2}}-\frac{2(4 N-1)}{3} \lambda^{\prime 2} \ln \frac{9 g^{2} v_{0}^{2}}{16 T^{2}}\right. \\
\left.-6 \lambda^{2} \ln \frac{N \lambda v_{0}^{2}}{3 T^{2}}+\lambda^{2} \frac{8(N+2)}{3}+\frac{27}{2} g^{4}+18 g^{2} \lambda\right] \\
+\frac{1}{2} A_{0}^{2}\left(\frac{N+4}{6} g^{2} T^{2}+\frac{N g^{2} m^{2}}{8 \pi^{2}}-\frac{N+4}{2 \pi^{2}} g^{2} \Lambda T\right)+\frac{g^{4}}{192 \pi^{2}} A_{0}^{4}+\frac{1}{8} g^{2} A_{0}^{2} \Phi_{0}^{2}
\end{gathered}
$$

$\left(\left\langle\Phi^{2}\right\rangle_{T=0}=N v_{0}^{2}\right)$. The large $\mathrm{N}$ form of this potential depends crucially on the way the different couplings scale with $\mathrm{N}$ :

$$
g^{2}=\frac{\tilde{g}^{2}}{N^{\alpha}}, \quad \lambda=\frac{\tilde{\lambda}}{N^{\beta}}, \quad m^{2}=\frac{\hat{m}^{2}}{N^{\gamma}} .
$$

By inspecting the second line of eq.(13) one realizes, following the argument of [13], that the stability of the symmetry breaking vacuum at $\mathrm{T}=0$ requires

$$
\frac{g^{4}}{\lambda} \leq \mathcal{O}\left(N^{0}\right) \quad \text { or } \quad \beta-2 \alpha \leq 0
$$

This restriction excludes the proposition for the large $\mathrm{N}$ scaling law suggested in [11] $(\beta=1, \alpha=1 / 3)$. We continue our analysis along the lines advocated in [6], which corresponds to

$$
\alpha=1, \quad \beta=2, \quad \gamma=1 .
$$


The length of the $\Phi_{0}$-field is assumed to be $\mathcal{O}\left(N^{1 / 2}\right)$. We also note that the choice $\alpha=1$ for the scaling of the gauge coupling agrees with similar prescriptions used for $S U\left(N_{c}\right)$ gauge theory coupled to $N_{f}$ fermions in the $N_{f} \rightarrow \infty$ limit [14.

Substituting these scaling relations into eq.(13), we obtain the following form of the leading large $\mathrm{N}$ potential:

$$
\begin{aligned}
U\left[A_{0}, \Phi_{0}\right]= & \frac{1}{2 N} \Phi_{0}^{2}\left[\tilde{m}^{2}\left(1-\frac{27 \tilde{g}^{4}}{64 \tilde{\lambda} \pi^{2}}\right)+\left(\frac{9 \tilde{g}^{2}}{4}+\frac{2 \tilde{\lambda}}{3}\right)\left(\frac{T^{2}}{12}-\frac{\Lambda T}{2 \pi^{2}}\right)\right] \\
& +\frac{1}{24 N^{2}} \Phi_{0}^{4}\left(\tilde{\lambda}+\frac{27 \tilde{g}^{4}}{128 \pi^{2}}\left(1-\ln \frac{\tilde{g}^{2} v_{0}^{2}}{4 T^{2}}\right)\right) \\
& \frac{1}{2} A_{0}^{2}\left(\frac{\tilde{g}^{2}}{6} T^{2}-\frac{\tilde{g}^{2}}{2 \pi^{2}} \Lambda T\right)+\frac{1}{8 N} \tilde{g}^{2} A_{0}^{2} \Phi_{0}^{2}
\end{aligned}
$$

This result clearly shows that under the scaling conditions of eq.(16) only the result of the gauge integration survives the large $\mathrm{N}$ limit. This conclusion agrees with the one obtained in Ref. [6] concerning the class of the leading Feynman graphs drawn in the full 4-d finite-T theory. Each term of the potential is $\mathcal{O}\left(N^{0}\right)$, that is the same order of magnitude as that of the gauge part of the 3-d effective action. The latter one becomes the copy of three effectively Abelian gauge fields because $g^{2}$ now is scaling with an inverse power of $\mathrm{N}$ [1]. The self-interaction of the 3 -d isovector scalars $A_{0}$ belongs also to the subleading terms of the potential, therefore its action becomes formally quadratic, too. With the (almost) usual 3-d-rescaling of the fields the leading $\mathrm{N}$ effective action is given by the following expression (the flavor index q has been omitted for convenience):

$$
\begin{gathered}
\frac{1}{2} \int d^{3} x\left\{U_{i}^{a}\left[\left(-\partial_{l}^{2}+\tilde{g}_{3}^{2} \phi^{+} \phi\right) \delta_{i j}+\left(1-\frac{1}{\alpha}\right) \partial_{i} \partial_{j}\right] U_{j}^{a}+2 i \tilde{g}_{3} \sqrt{N} U_{i}^{a} J_{i}^{a}\right. \\
\left.U_{0}^{a}\left[-\partial_{l}^{2}+\frac{\tilde{g}_{3}^{2} T}{6}+\frac{\tilde{g}_{3}^{2} \phi^{+} \phi}{4}\right] U_{0}^{a}+\phi^{+}\left[-N \partial_{l}^{2}+\hat{m}^{2}\right] \phi+\frac{\hat{\lambda}_{3}}{12}\left(\phi^{+} \phi\right)^{2}\right\} \\
+(3-\mathrm{d})-\text { counterterms }
\end{gathered}
$$

with

$$
\begin{gathered}
J_{i}^{a}=\frac{1}{4}\left(\partial_{i} \phi_{q}^{+} \tau^{a} \phi_{q}-\phi_{q}^{+} \tau^{a} \partial_{i} \phi_{q}\right), \\
\hat{m}^{2}=\tilde{m}^{2}\left(1-\frac{27 \tilde{g}_{3}^{4}}{64 \tilde{\lambda}_{3} \pi^{2}}\right)+\left(\frac{9 \tilde{g}_{3}^{2}}{4}+\frac{2 \tilde{\lambda}_{3}}{3}\right) \frac{T}{12}, \quad \hat{\lambda}_{3}=\tilde{\lambda}_{3}+\frac{27 \tilde{g}^{4}}{128 \pi^{2}}\left(1-\ln \frac{\tilde{g}^{2} v_{0}^{2}}{4 T^{2}}\right), \\
\tilde{g}_{3}^{2}=\tilde{g}^{2} T, \quad \tilde{\lambda}_{3}=\tilde{\lambda} T, \quad U_{i}^{a}=A_{i}^{a} / \sqrt{T}, \quad U_{0}^{a}=A_{0}^{a} / \sqrt{T}, \quad \phi=\sqrt{\frac{1}{N T}} \Phi_{0} .
\end{gathered}
$$

The integration over $U_{i}$ and $U_{0}$ can be performed trivially. For instance in the Landau-gauge the non-regularised contributions to the effective $\phi$-action 
reads:

$$
3 \operatorname{Tr} \log \left(-\partial_{l}^{2}+\tilde{g}_{3}^{2} \phi^{+} \phi\right)+\frac{3}{2} \operatorname{Tr} \log \left(-\partial_{l}^{2}+\frac{\tilde{g}_{3}^{2} T}{6}+\frac{\tilde{g}_{3}^{2} \phi^{+} \phi}{4}\right)+\frac{N}{2} \tilde{g}_{3}^{2} J_{i}^{a} D_{i j}^{a b} J_{j}^{b},
$$

where $D_{i j}^{a b}$ denotes the propagator of the transversal gauge quanta on the scalar background. The last term is actually a correction to the kinetic term of the Higgs-fields. Taking into account that the fluctuations of $\phi$ are $\mathcal{O}(1 / \sqrt{N})$, the magnitude of this term is also unity. After performing the 3 -d functional traces the 3-d mass-counterterms proportional to the gauge coupling exactly cancel and the following effective scalar theory is derived:

$$
\begin{aligned}
S_{3 d}[\phi] & =\int d^{3} x\left\{\frac{1}{2} \phi^{+}\left[-N \partial_{l}^{2}+\hat{m}^{2}\right] \phi+\frac{1}{2} N \tilde{g}_{3}^{2} J_{i}^{a} D_{i j}^{a b} J_{j}^{b}+\frac{\hat{\lambda}_{3}}{24}\left(\phi^{+} \phi\right)^{2}\right. \\
& \left.-\frac{1}{4 \pi}\left(\tilde{m}_{A}^{2}+\frac{\tilde{g}_{3}^{2} \phi^{+} \phi}{4}\right)^{3 / 2}-\frac{\tilde{g}_{3}^{3}}{8 \pi}\left(\frac{T}{6}+\frac{\phi^{+} \phi}{4}\right)^{3 / 2}-\frac{\tilde{\lambda}_{3} \Lambda}{6 \pi^{2}} \phi^{+} \phi\right\} .
\end{aligned}
$$

In this equation we indicate, where could have appeared the magnetic screening mass $\tilde{m}_{A}^{2}$, which to leading order in $\mathrm{N}$ is strictly 0 .

The structure of the effective theory eq.(21) agrees with the basic structure of the scalar theory to be investigated in later parts of this paper, up to the correction of the kinetic part which we shall omit from our quantitative investigation.

\subsection{The Effective Model for $N=1$}

For $N=1$ one has to go back to the form of the potential term of the 3 - $d$ effective action given in eq.(13). This form has been established in Ref. [12], where also the 1-loop solution of the 3-d effective model has been extensively discussed. The authors suggest the existence of an interesting screening mass hierarchy at the temperature of the phase transition, whose characterisation depends crucially on the value of $m_{H}(T=0)$.

Namely, the effective screening mass squares of the $A_{0}$ and $A_{i}$ quanta given by the formulae

$$
m_{D}^{2}=\frac{5}{6} g^{2} T^{2}+\frac{m^{2} g^{2}}{8 \pi^{2}}+\frac{g^{2}}{4} \Phi_{0}^{2}\left(T=T_{c}\right), \quad m_{W}^{2}=\frac{g^{2}}{4} \Phi_{0}^{2}\left(T=T_{c}\right)
$$

turn out to be a factor 4-6 larger than that of the scalar quanta for $m_{H}(T=$ $0)=35 \mathrm{GeV}$. This ratio monotonically increases as one goes to smaller Higgs 
mass values, its value is $\sim 25$ for $m_{H}(T=0)=20 \mathrm{GeV}$. Therefore, at least in this region, the integration over these very massive degrees of freedom seems to be well-founded.

After gaussian integration and after 3-d mass-renormalisation of $A_{0}$ the following effective action for the Higgs-field is obtained:

$$
\begin{gathered}
S_{3 d}[\phi]=\int d^{3} x\left[\frac{1}{2} \partial_{l} \phi^{+} \partial_{l} \phi+\frac{1}{2} \hat{M}_{\phi}^{2} \phi^{+} \phi+\frac{\hat{\lambda} T}{24}\left(\phi^{+} \phi\right)^{2}\right. \\
\left.-\frac{g_{3}^{3}}{32 \pi}\left(\frac{4 m_{D}^{2}}{g_{3}^{2}}+\phi^{+} \phi\right)^{3 / 2}-\frac{g_{3}^{3}}{16 \pi}\left(\frac{4 m_{A}^{2}}{g_{3}^{2}}+\phi^{+} \phi\right)^{3 / 2}\right]
\end{gathered}
$$

with the meaning of the notations given below (not to be mixed with previous meaning of some of them!):

$$
\begin{gathered}
\hat{M}_{\phi}^{2}=\hat{m}^{2}+\left(\frac{3 g^{2}}{16}+\frac{\lambda}{12}\right) T^{2}-\lambda \frac{T \Lambda}{2 \pi^{2}} \\
\hat{m}^{2}=m^{2}\left\{1-\frac{1}{32 \pi^{2}}\left[\left(\frac{9}{2} g^{2}+\lambda\right) \ln \frac{3 g^{2} v_{0}^{2}}{4 T^{2}}+\lambda \ln \frac{\lambda v_{0}^{2}}{3 T^{2}}\right]-\frac{1}{128 \pi^{2}}\left(45 g^{2}+20 \lambda+\frac{27 g^{4}}{\lambda}\right)\right\} \\
\hat{\lambda}=\lambda-\frac{9}{16 \pi^{2}}\left(\frac{9 g^{4}}{16}+\frac{3 g^{2} \lambda}{4}+\frac{\lambda^{2}}{3}\right) \\
-\frac{3}{8 \pi^{2}}\left\{g^{4}\left(\frac{3}{8} \ln \frac{g^{2} v_{0}^{2}}{4 T^{2}}-\frac{3}{2} \ln \frac{g^{2} v_{0}^{2}}{\sqrt{2} T^{2}}\right)+\frac{\lambda^{2}}{4} \ln \frac{\lambda v_{0}^{2}}{3 T^{2}}+3\left(\frac{3 g^{2}}{4}+\frac{\lambda}{6}\right)^{2} \ln \frac{3 g^{2} v_{0}^{2}}{4 T^{2}}\right\}
\end{gathered}
$$

Eqs. 23)-(25) represent the version of the effective scalar model, which we are going to employ in the non-perturbative investigations of the next sections. Its general structure fully coincides with the result of the large $\mathrm{N}$ calculation, providing some extra argument for its relevance.

For its discrete treatment it is most convenient to keep $g^{2}$ fixed and to think of the effective action as being defined by the couplings $\hat{M}_{\phi}$ and $\hat{\lambda}$. After the performing the phase transition analysis one uses equations eq.(24) and eq.(25) for finding a posteriori the true couplings (or what is equivalent the Higgs mass). The deviation of the quantities with "hat" from "hatless" partners depends crucially on the logarithmic terms appearing in eq.(24) and eq.(25). With decreasing Higgs-mass they become increasingly important, making our conclusions for this region sensitive to the renormalisation scheme, implemented in the 1-loop calculations. For comparison we can consider a choice of the renormalisation scale in eq.(10), where the logarithmic terms are missing $(\mu=T)$. (The assumption of unchanged tree-level relationship between the couplings and the W and Higgs masses is maintained 
somewhat arbitrarily.) The results for $T_{c}$ and $\frac{\Phi_{c}}{T_{c}}$ will be seen in the next section to agree with their values found in the Linde scheme within 10-15\% for $m_{H}(T=0)=35 \mathrm{GeV}$ when the mean field solution method is applied. The deviation sharply increases for smaller values of the Higgs mass.

\section{Lattice Formulation and Mean Field Anal- ysis of the Effective Scalar Model}

The discretisation of eq.(23) proceeds by introducing the dimensionless field

$$
\psi=\sqrt{a} \phi
$$

with $a$ being the lattice constant. Introducing also dimensionless masses and temperature via the relations:

$$
2 m_{A} a=\sqrt{\gamma_{M}} g \Theta, \quad 2 m_{D} a=\sqrt{\gamma_{E}} g \Theta, \quad \Theta=a T
$$

one finds

$$
\begin{aligned}
S_{3 d, l a t} & =\sum_{x}\left[\frac{1}{2 \kappa} \psi_{x}^{+} \psi_{x}+\frac{\hat{\lambda} \Theta}{24}\left(\psi_{x}^{+} \psi_{x}\right)^{2}-\frac{1}{2} \sum_{e}\left(\psi_{x+e}^{+} \psi_{x}+\psi_{x}^{+} \psi_{x+e}\right)\right. \\
& \left.-\frac{g^{3} \Theta^{3 / 2}}{32 \pi}\left(2\left(\gamma_{M} \Theta+\psi_{x}^{+} \psi_{x}\right)^{3 / 2}+\left(\gamma_{E} \Theta+\psi_{x}^{+} \psi_{x}\right)^{3 / 2}\right)\right]
\end{aligned}
$$

with

$$
\frac{1}{2 \kappa}=\frac{1}{2} \hat{m}^{2} a^{2}+\frac{1}{2}\left(\frac{3}{16} g^{2}+\frac{\lambda}{12}\right) \Theta^{2}-\frac{C}{2} \Theta \Sigma\left(L^{3}\right)+3 .
$$

Here $\Sigma\left(L^{3}\right)$ denotes the lattice regularized version of the linearly divergent integral $\int d^{3} p /\left(8 \pi^{3} p^{2}\right)$, giving rise to the linear divergence in eq.(24). The perturbative values of the constants $C$ and $\gamma_{E}$ are given by $C=\lambda$ and

$\gamma_{E}=10 / 3$. We emphasize once more that the input parameters into the lattice calculation are $\kappa, \hat{\lambda}$ and $\mathrm{g}$.

For each fixed value of $\Theta$ the discrete theory, eq.(28), will pass through a phase transition at a certain value $\kappa_{c}(\Theta)$ or

$$
Z_{c}(\Theta)=\left(\frac{1}{2 \kappa_{c}(\Theta)}-3+\frac{C}{2} \Sigma\left(L^{3}\right) \Theta\right) \frac{1}{\Theta^{2}} .
$$

The continuum limit is defined by the limit $\Theta \rightarrow 0$ of $Z_{c}(\Theta)$ providing a non-trivial value $Z_{0}$. 
When the value of $Z_{0}$ has been determined, eq.(29) is rewritten as a relation between the unknown physical value of $T_{c}$ and $\lambda$ :

$$
\frac{\hat{m}^{2}}{2 T_{c}^{2}}=Z_{0}-\frac{1}{2}\left(\frac{3}{16} g^{2}+\frac{\lambda}{12}\right) .
$$

In eq.(31) $\hat{m}$ is replaced by the expression given in eq.(25). The other relation is provided by the definition of $\hat{\lambda}$ in eq.(25). From these two equations the physical values of the Higgs mass and of the critical temperature can be determined.

In concrete terms, after introducing the dimensionless temperature (in units of the $\mathrm{T}=0$ vacuum expectation value $v_{0}$ ) the following two equations are to be solved (for instance, iteratively):

$$
\begin{gathered}
\tau_{c}^{2}=\frac{\lambda}{6} \frac{1}{\frac{3}{16} g^{2}+\frac{\lambda}{12}-2 Z_{0}(\hat{\lambda})}\left(1-f_{1}\left(\lambda, \tau_{c}\right)\right), \\
\lambda=\hat{\lambda}+f_{2}\left(\lambda, \tau_{c}\right)
\end{gathered}
$$

with the functions

$$
\begin{gathered}
f_{1}\left(\lambda, \tau_{c}\right)=\frac{1}{128 \pi^{2}}\left(45 g^{2}+20 \lambda+\frac{27 g^{4}}{\lambda}\right)+\frac{1}{32 \pi^{2}}\left[\left(\frac{9}{2} g^{2}+\lambda\right) \ln \frac{3 g^{2}}{4 \tau_{c}^{2}}+\lambda \ln \frac{\lambda}{3 \tau_{c}^{2}}\right], \\
f_{2}\left(\lambda, \tau_{c}\right)=\frac{9}{16 \pi^{2}}\left(\frac{9 g^{4}}{16}+\frac{3 g^{2} \lambda}{4}+\frac{\lambda^{2}}{3}\right) \\
+\frac{3}{8 \pi^{2}}\left[g^{4}\left(\frac{3}{8} \ln \frac{g^{2}}{4 \tau_{c}^{2}}-\frac{3}{2} \ln \frac{g^{2}}{\sqrt{2} \tau_{c}^{2}}\right)+\frac{\lambda^{2}}{4} \ln \frac{\lambda}{3 \tau_{c}^{2}}+3\left(\frac{3 g^{2}}{4}+\frac{\lambda}{6}\right)^{2} \ln \frac{3 g^{2}}{4 \tau_{c}^{2}}\right] .
\end{gathered}
$$

(Here the relation $m^{2}=-\lambda v_{0}^{2} / 6$ has been used again.)

Having found $\lambda$ and $\tau_{c}$ the physical Higgs-mass and the critical temperature are determined easily from the relations:

$$
\tau_{c}^{2}=\frac{T_{c}^{2}}{v_{0}^{2}}, \quad \lambda=\frac{3 m_{H}^{2}}{v_{0}^{2}} .
$$

The simplest illustration of a non-trivial continuum limit $(Z \neq 0)$ is given by the mean field analysis of the system eq.(28). The mean-field expression of the free energy density as a function of the mean field order parameter $s$ is

$$
F[s]=\left(\frac{1}{2 \kappa}-d\right) s^{2}+\frac{\Theta \hat{\lambda}}{24} s^{4}-\frac{g^{3} \Theta^{3}}{32 \pi}\left[2\left(\gamma_{M}+\frac{s^{2}}{\Theta}\right)^{3 / 2}+\left(\gamma_{E}+\frac{s^{2}}{\Theta}\right)^{3 / 2}\right] .
$$


The condition for the existence of a non-trivial $\left(s_{0} \neq 0\right)$ minimum is written as

$$
\left(\frac{1}{2 \kappa}-d\right) \frac{1}{\Theta^{2}}+\frac{\hat{\lambda}}{12} \frac{s_{0}^{2}}{\Theta}-\frac{3 g^{3}}{32 \pi}\left[\left(\gamma_{M}+\frac{s_{0}^{2}}{\Theta}\right)^{1 / 2}+\frac{1}{2}\left(\gamma_{E}+\frac{s_{0}^{2}}{\Theta}\right)^{1 / 2}\right]=0 .
$$

Introducing the scaled free energy $F[s] / \Theta^{3}$ and the scaled order parameter $\bar{s}^{2}=s^{2} / \Theta$, one can eliminate the common term $\left(\frac{1}{2 \kappa}-d\right) \frac{1}{\Theta^{2}}$ from the degeneracy condition for the free energy minima and determine the position of the degenerate symmetry breaking minimum $\bar{s}_{0}$ from the equation

$$
\begin{gathered}
-\frac{\hat{\lambda}}{24} \bar{s}_{0}^{4}+\frac{3 g^{3} \bar{s}_{0}^{2}}{32 \pi}\left[\left(\gamma_{M}+\bar{s}_{0}^{2}\right)^{1 / 2}+\frac{1}{2}\left(\gamma_{E}+\bar{s}_{0}^{2}\right)^{1 / 2}\right] \\
-\frac{g^{3}}{32 \pi}\left[2\left(\gamma_{M}+\bar{s}_{0}^{2}\right)^{3 / 2}+\left(\gamma_{E}+\bar{s}_{0}^{2}\right)^{3 / 2}\right]+\frac{g^{3}}{32 \pi}\left(2 \gamma_{M}^{3 / 2}+\gamma_{E}^{3 / 2}\right)=0 .
\end{gathered}
$$

The quantity $Z_{c, m f}$ is calculated as

$$
Z_{c, m f}=\left(\frac{1}{2 \kappa_{c}}-d\right) / \Theta^{2}=-\frac{\hat{\lambda}}{12} \bar{s}_{0}^{2}+\frac{3 g^{3}}{32 \pi}\left[\left(\gamma_{M}+\bar{s}_{0}^{2}\right)^{1 / 2}+\frac{1}{2}\left(\gamma_{E}+\bar{s}_{0}^{2}\right)^{1 / 2}\right] .
$$

We note that the right hand side is independent of $\Theta$ and therefore $Z_{0, m f} \equiv$ $Z_{c, m f}$. In Fig. 1) we show the mean field estimate of the quantity $Z$ for $\gamma_{B}=0,1,2$ (see eq.(41) for the meaning of $\gamma_{B}$ ) as a function of $\hat{m}_{H}$. The rapid rise for small $\hat{m}_{H}$ leads uniformly (independent of $\gamma_{B}$ ) to a "critical" $\hat{\lambda}$ at which the right hand side of eq.(31) vanishes. In the approximation $m^{2}=\hat{m}^{2}$ and $\lambda=\hat{\lambda}$ this corresponds to a divergent critical temperature. However, because of the uncertainties due to the application of different renormalization conditions this phenomenon might be an artefact of the above approximation. The data point appearing in Fig. 1) is the result of our numerical simulation. The analysis leading to it is described in section 4 .

The order parameter discontinuity characterising the phase transition can be expressed with help of $\bar{s}_{0}$ as

$$
\Phi\left(T_{c}\right)=\bar{s}_{0} T_{c}
$$

A very contentful characterisation of the first order transition is given by the surface tension, $\sigma$, between coexisting ordered and disordered regions. 
Exploiting the scaling behavior of the mean field solution one finds for it in the thin wall approximation:

$$
\begin{gathered}
\bar{\sigma}_{m f}=\frac{\sigma}{T_{c}^{3}}=\int_{0}^{\bar{s}_{0}} d \bar{s}[2 f(\bar{s})]^{1 / 2} \\
f(\bar{s})=\frac{F(s)-F(0)}{\Theta^{3}}=Z_{0, m f} \bar{s}^{2}+\frac{\lambda}{24} \bar{s}^{4}-\frac{g^{3}}{32 \pi}\left[2\left(\gamma_{M}+\bar{s}^{2}\right)^{3 / 2}+\left(\gamma_{E}+\bar{s}^{2}\right)^{3 / 2}\right] \\
+\frac{g^{3}}{32 \pi}\left(2 \gamma_{M}^{3 / 2}+\gamma_{E}^{3 / 2}\right) .
\end{gathered}
$$

In Fig. 2) we show the true Higgs mass square as a function of $\hat{m}_{H}^{2}=$ $\hat{\lambda} v_{0}^{2} / 3$. The trivial linear relation goes over into a strongly non-linear functional form only for Higgs mass values less than $25 \mathrm{GeV}$. For larger values $m_{H}$ and $\hat{m}_{H}$ are seen to agree within $10 \%$. In Fig. 3) $T_{c}$ is shown as a function of $m_{H}$ and $\hat{m}_{H}$. Below $m_{H}=25 \mathrm{GeV} T_{c}$ is rising with decreasing Higgs mass, reflecting the approach to zero of the denominator in the expression of $\tau_{c}$ in eq. (32).

The solid curve in this figure shows the result of the approximation $m_{H}=\hat{m}_{H}, \lambda=\hat{\lambda}$. This approximation corresponds to the choice of normalisation scale $\mu=T$ in the interpretation of eq.(10). It leads to the definition of the continuum limit directly from eq.(31) avoiding the complicated procedure of solving eq.(32) and eq.(33). This approximation for a Higgs mass near $m_{H}=15 \mathrm{GeV}$ would indicate infinite $T_{c}$, which would hint to the non-restorability of the broken gauge symmetry for Higgs masses smaller than this limiting value. In principle, the non-restoration of the symmetry even for infinite temperature is not excluded and there are known examples of such behaviour due to quantum corrections to the effective potential [15]. These are the logarithmic terms of the expression of $\hat{\lambda}$ and $\hat{m}^{2}$, which counterbalance the increasing tendency of $T_{c}$, when the Linde-type renormalisation condition is being used. However, large logarithmic corrections due to the essential deviation of the relevant range of the temperature from the chosen renormalisation scale make the 1-loop approximation unreliable. The difference in the two curves of Fig. 3) for $m_{H}<30 \mathrm{GeV}$ indicates the importance of higher loop contributions in the equations governing the continuum limit.

In Fig. 4) the dependence of the order parameter discontinuities on the strength of magnetic screening is shown as a function of $\hat{m}_{H}$. For nonvanishing magnetic mass the first order transition ends in a tricritical point (TCP). Following [2] we parametrize the amount of magnetic screening in proportion to the value obtained from a selfconsistent Dyson-Schwinger equa- 
tion:

$$
m_{A}=\gamma_{B} \frac{g^{2} T}{3 \pi} .
$$

The mean field analysis yields then for the end point values of the Higgsmasses:

$$
\begin{array}{ll}
\gamma_{B}=1, & m_{H}(T C P)=84.5 \mathrm{GeV} \\
\gamma_{B}=2 & , \quad m_{H}(T C P)=61.7 \mathrm{GeV}
\end{array}
$$

in good agreement with the continuum perturbative estimates. In the insertion of Fig. 4) the uncertainty in the order parameter discontinuity resulting from the use different renormalisation schemes is illustrated. The application of the Linde-conditions leads for small Higgs masses (dotted line) to harder transitions. We can conclude, that in the region $m_{H}<30-35 \mathrm{GeV}$ no reliable continuum statement can be made on the basis of the 1-loop calculation.

Finally we display in Fig. 5) as function of $\hat{m}_{H}$ the mean field result for the interface tension eq.(40).

\section{Numerical Simulation and Results}

We have simulated the lattice model eq.(28) on hypercubic lattices with linear extent $L$ and periodic boundary conditions. The electric and magnetic screening mass parameters were choosen to be $\gamma_{E}=10 / 3$ and $\gamma_{B}=1$, eq. (41). At values for the $\mathrm{W}$-mass $m_{W}=80 \mathrm{GeV}$ and for the zero temperature vacuum expectation value of the Higgs field $v_{0}=246 \mathrm{GeV}$ the gauge coupling constant $g$ of eq.(28) is determined by the tree level relation eq.(22) applied at $T=0$. These choices first leave us with 3 free parameters of the theory $\hat{\lambda}, \Theta$ and the hopping parameter $\kappa$. Choosing furthermore a value for the bare quartic coupling $\hat{\lambda}$ or correspondingly via eqs.(32, 33, 34 a physical value for the Higgs mass $m_{H}$, we are left in our simulation with a two parameter theory. In the $\kappa-\Theta$ plane of couplings we expect the symmetry restoration phase transition whose properties we would like to study. In detail we shall discuss how to extract $T_{c}$, the order parameter discontinuity, $\Phi\left(T_{c}\right)$, the latent heat $\mathcal{L}$ and the interface tension $\sigma$ from the raw data. 


\subsection{Lattice Observables and the Continuum Limit}

Both bare parameters $\kappa$ and $\Theta$ serve the purpose of constructing the continuum limit. In particular we will be interested in the study of various quantities measured at $T_{c}$. We thus will tune the hopping parameter to the critical line $\kappa_{c}(\Theta)$ and consider the limit $\Theta \rightarrow 0$, which at the same time removes the lattice cutoff $a$. In this limit the theory turns into the 3-dimensional Gaussian model. From the $\Theta$-dependence of the critical hopping parameter, we can determine the continuum limit of the quantity $Z_{c}(\Theta)$, which has been defined in eq.(30). With the help of eqs.(32, 33, 34) this fixes the critical temperature in units of the Higgs mass. We furthermore study various operators, which allow the extraction of physically relevant quantities such as the order parameter discontinuity at $T_{c}$, the surface tension and the latent heat. In particular we consider the following observables

$O_{1}=\frac{1}{L^{3}} \sum_{x} \psi_{x}^{+} \psi_{x}, O_{2}=\frac{1}{3 L^{3}} \sum_{x, e} \psi_{x}^{+} \psi_{x+e}, \quad O_{3}=\frac{1}{L^{3}} \sqrt{\left\{\left(\sum_{x} \psi_{x}\right)^{+}\left(\sum_{y} \psi_{y}\right)\right\}}$,

whose distributions $P\left(O_{i}\right)$, expectation values $\left\langle O_{i}\right\rangle$ and diagonal fluctuations $C\left(O_{i}\right)=L^{3}\left\langle O_{i}^{2}-\left\langle O_{i}\right\rangle^{2}\right\rangle$ for $i=1,2,3$ are determined in the simulation. $O_{1}$ corresponds to the average length square of the Higgs field, $\mathrm{O}_{2}$ to a part of the kinetic term of the action, while $\mathrm{O}_{3}$ corresponds to the average field. They can be related to the corresponding continuum observables through relations discussed in the previous sections. Here we note that the lattice fields $\psi$ and the continuum fields $\Phi$ differ by a factor of $\sqrt{T}$. In the limit $\Theta \rightarrow 0$ we thus expect appropriately scaled dimensionless ratios to reach constant values. For instance we examine the ratio $\frac{\left\langle O_{3}\right\rangle}{\Theta^{1 / 2}}$, which in the limit $\Theta \rightarrow 0$ converges to the continuum result $\Phi\left(T_{c}\right) / T_{c}$. Of course we have to investigate here carefully possible scaling violations due to finite lattice spacing $a$, as well as finite volume effects due to the finite extent $L^{3}$ of the lattices. These problems will be discussed in detail in connection with the numerical analysis of the various observables.

In general we expect that with decreasing values of $\Theta$ (decreasing lattice spacing) it will be increasingly difficult investigate the properties of finite temperature phase transition as any signal for a possible first order phase transition will become small in units of the lattice spacing. Moreover, we have to understand the possible range of values of the Higgs mass, for which we can study the phase structure of our model. For large values of the Higgs 
mass the transition is expected to be at best weakly first order, which then requires large lattices to resolve any discontinuity in physical observables. In order to explore the parameter range, in which we can perform statistically significant studies of our model we have studied thermal cycles across the phase transition line $\kappa_{c}(\Theta)$ varying also the Higgs mass parameter.

In Fig. 6) and Fig. 7) we display the results of thermal cycles for the order parameter $\left\langle O_{3}\right\rangle$ on $18^{3}$ lattices with a statistics of 4000 sweeps per data point. Fig. 6) displays results at fixed values of $\Theta=5$. The parameter $\hat{m}_{H}$ ranges from $\hat{m}_{H}=35 \mathrm{GeV}$ to $\hat{m}_{H}=47 \mathrm{GeV}$ in this figure. The broad and pronounced hysteresis at small values of $\hat{m}_{H}$ rapidly shrinks with increasing $\hat{m}_{H}$ and disappears at $\hat{m}_{H}=47 \mathrm{GeV}$. We have included into both figures the mean field result for the critical point (dashed vertical lines) and the mean field value for the order parameter discontinuity (solid circles and height of the dashed lines). While the locations of the phase transitions roughly agree with the centers of the hystereses, Fig. 6) indicates a more rapid decrease of the order parameter jump as compared to the mean field result. At $\hat{m}_{H}=47$ $\mathrm{GeV}$ and $\Theta=5$ it is presumably notoriously difficult to determine infinite volume values of possible gaps in operators, or to determine whether the phase tranition is discontinuous at all. This is the main reason why we have decided to choose the value $\hat{m}_{H}=35 \mathrm{GeV}$ for a detailed and high precision study of the symmetry restoration phase transition. This value of $\hat{m}_{H}=35$ $\mathrm{GeV}$ is still large enough for not being plagued too much in the continuum interpretation by the ambiguities of different renormalization schemes. It corresponds to a physical value of the Higgs mass of $m_{H}=37.16 \mathrm{GeV}$, see Fig. 2).

Fig. 7) displays at the selected value of $\hat{m}_{H}=35 \mathrm{GeV}$ the variation of the hysteresis of the order parameter $\mathrm{O}_{3}$ as a function of $\Theta$. As expected the first order signal weakens with decreasing $\Theta$. Comparing the mean field values of the order parameter jump with the $\mathrm{MC}$ data we again note a more rapid weakening of the first order signal. From these data and from additional MC-runs we expect that the interesting region in which we might be able to resolve possible first order signals on the given sized lattices extends down to $\Theta$-values as low as $\Theta=1$. The typical lattices sizes, which we use in our simulation range from $8^{3}$ up to $18^{3}$. Typical statistics for simulations on any of the lattice sizes is about $10^{6}$ sweeps. To overcome the problem of large tunneling times at the first order phase transition, we have used the multicanonical ensemble approach [16]. We see later that even at $\Theta=3$ 
the phase transition appears to be rather week, e.g. double peak structures in the probability distributions $P\left(O_{i}\right)$ at the transition point are not very pronounced.

\subsection{The critical Temperature $T_{c}$}

The perturbative treatment of the effective model predicts a certain shape of the phase transition line $\kappa_{c}(\Theta)$, provided $Z_{c}(\Theta)$ of eq. (30) is known. However the mean field treatment of the theory predicts that $Z_{c}(\Theta) \equiv Z_{0, m f}$ is just a constant independent of $\Theta$. We therefore might expect that in a first approximation also the fully fluctuating theory can be described with a constant $Z_{c}(\Theta)=Z_{0, m c}$ for small values of $\Theta$. At $\hat{m}_{H}=35 \mathrm{GeV}$ we estimate the critical hopping parameter from thermal cycles. These are displayed in Fig. 8) for $\Theta$ less than 1. We observe a clear bending of the phase transition line towards smaller $\kappa_{c}$-values at $\Theta$-values of about unity. Thus the numerical data predict a nonzero and positive value for $Z_{0, m c}$. A fit to the shape of the phase transition line according to eq. (30) then results into a Monte Carlo determination of the quantity $Z_{0, m c}=0.0187(16)$, which is to be compared with the mean field value of $Z_{0, m f}=0.0113$ at same values of couplings. Noticeably our data are consistent with the presence of a linear $\Theta$-dependent term in eq.(30) with $C=0.93(16) \times \lambda$, as it is predicted by perturbation theory (the continuous curve in the figure). The mean field phase transition line (dashed curve) deviates from the $\mathrm{MC}$ data, which at

small $\Theta$ slightly overshoot the $\kappa$-value $\kappa=\frac{1}{6}$. At the given value of $Z_{0, m c}$ we may now determine the critical temperature for both of our renormalization schemes, namely in the $\mu=T$ scheme (without logarithmic corrections), as well as in the Linde-scheme, which incorporates them. The result is

$$
\begin{array}{ll}
T_{c}=114.9(36) \mathrm{GeV} & (\mu=T) \\
T_{c}=114.3(30) \mathrm{GeV} & \text { Linde scheme. }
\end{array}
$$

Both numbers are displayed in Fig. 3), the triangle corresponds to the $\mu=T$ scheme and is plotted at its value of $\hat{m}_{H}$ while the circle corresponds to the renormalization scheme with logarithmic corrections plotted at the the physical Higgs mass value $m_{H}$. It is noticeable that the difference between the schemes is very small. These Monte Carlo determinations of $T_{c}$ should be compared with the mean field result $T_{c}=99.6 \mathrm{GeV}$. 


\subsection{Critical Hopping Parameters}

For the value of $\hat{m}_{H}=35 \mathrm{GeV}$, we have performed a detailed finite size scaling analysis of the symmetry restoration phase transition at three values of $\Theta$, namely $\Theta=3,4$ and 5. In Fig. 9) we compare the results of a thermal cycle on an $18^{3}$ lattice for the operator $\left\langle O_{1}\right\rangle$, with results of multicanonical simulations for the same operator on $8^{3}, 10^{3}$ and $12^{3}$ lattices. The mean field position of the critical point, as well as its true infinite volume value, deduced from finite size scaling analysis, are indicated by the vertical dashed lines in the figure. Defining pseudocritical points $\kappa_{\max }(L)$ from the peak positions of the maxima of the diagonal fluctuation $C\left(O_{1}\right)$, see Fig. 10), the pseudocritical $\kappa$-values are extrapolated to infinite volume via

$$
\kappa_{\max }(L)=\kappa_{c}+\frac{b}{L^{3}}
$$

Fig. 11) shows at $\Theta=4$ the finite size scaling analysis leading to the infinite volume critical point. It is clear from these figures that multicanonical ensemble simulations combined with finite size scaling theory allow a precise determination of the location of the phase transition or discontinuities. They are in fact superior to standard methods e.g., thermal cycles, which even on a large $18^{3}$ lattice cannot produce numbers with comparable precision, see Fig. 9). Table 1) then contains for the selected $\Theta$-values a comparison of the mean field critical hopping parameters to the Monte Carlo results. They are very close and their difference is $\mathcal{O}\left(10^{-4}\right)$. As one might have expected the location of the phase transition at large values of $\Theta$ is well described by mean field behavior. This has to be contrasted with the observed behavior of $\kappa_{c}(\Theta)$ at values of $\Theta<1$, where the mean field approximation is not adequate.

\subsection{Determination of Discontinuities}

We obtain the infinite volume discontinuities in thermodynamic quantities by analyzing the volume dependence of probability distribution functions $P\left(O_{i}\right)$ of operators $O_{i}$ defined in eq. (43). In the vicinity of the transition they develop double peak structures. We determine the values of the couplings, where in the finite volume system the two peaks in $P\left(O_{i}\right)$ are of equal height. This defines e.g., at fixed value of $\Theta$, a value of the hopping parameter $\kappa_{e h}(L)$. Fits to the maxima of the distribution functions then yield finite volume 
Table 1: The critical hopping parameters $\kappa_{c}(\Theta)$, to the left: mean field results, to the right: MC results.

\begin{tabular}{||c|c|c||}
\hline$\Theta$ & $\kappa_{c}(\Theta)_{m f}$ & $\kappa_{c}(\Theta)_{m c}$ \\
\hline 3 & 0.161188 & $0.161112(4)$ \\
\hline 4 & 0.157170 & $0.157065(5)$ \\
\hline 5 & 0.152283 & $0.152170(2)$ \\
\hline
\end{tabular}

estimators for the values of operators in the metastable states, denoted by $\left\langle O_{i}\right\rangle_{S B}(L)$ (symmetry broken) and $\left\langle O_{i}\right\rangle_{S}(L)$ (symmetric) for $i=1,2,3$. The discontinuities $\Delta\left\langle O_{i}\right\rangle(L)=\left\langle O_{i}\right\rangle_{S B}(L)-\left\langle O_{i}\right\rangle_{S}(L)$ are then extrapolated to infinite volume using standard finite size scaling arguments [17]. Their large volume $L$-dependence is expected to scale with the volume,

$$
\Delta\left\langle O_{i}\right\rangle(L)=\Delta\left\langle O_{i}\right\rangle+\frac{a}{L^{3}} \quad i=1,2,3 .
$$

In Fig. 12) we display on $10^{3}$ lattices probability distributions of the operator $O_{1}$ i.e., the average length square of the scalar field at $\kappa_{e h}(L)$ for $\Theta$ values $\Theta=3,4$ and 5. As can be seen one finds clear double peak distribution functions, which can actually be observed for all of our operators $O_{i} i=$ $1,2,3$. Remarkably, however, the value of the minimum of the distribution functions rapidly rises with decreasing $\Theta$. At $\Theta=3$ the suppression of mixed phase states is hardly noticeable, which makes the phase transition a rather weak first order phase transition already.

Fig. 13) shows the finite size scaling analysis of $\left\langle O_{3}\right\rangle_{S B}(L)$ corresponding to the order parameter jump in the symmetry broken phase. A fit to the data with the form eq. (46) readily gives the infinite volume discontinuity $\Delta\left\langle\mathrm{O}_{3}\right\rangle$. Fig. 14) shows the same kind of analysis for the operator $O_{1}$, corresponding to the average length square of the scalar field. In this case $\left\langle O_{1}\right\rangle_{S}(L)$ also has to be considered for the calculation of the corresponding discontinuity. Carrying out the analysis for all our operators we arrive in Table 2) at the infinite volume values for the discontinuities for all considered operators and $\Theta$-values. They can be compared with the mean field order parameter jump which appears also in Table 2). In Table 2) we present the discontinuities in $O_{1}$ and $O_{2}$ scaled by the square of the discontinuity in $O_{3}$, the order 
parameter. They are consistent with a value of 1 . Thus actually the various discontinuities are not independent at the transition, but trivially connected. This is exactly what one expects from a mean field type of behavior at the transition.

The figures also exhibit some unusual finite size scaling behavior e.g., it can be noted that with increasing lattice size the finite volume estimators of the discontinuities increase. Many of the first order phase transitions in statistical physics and lattice gauge theories exhibit an opposite behavior, that is with increasing lattice size the values of the obtained discontinuities decrease. Figs. 13) and 14) also indicate that finite volume corrections become rather large in size at the smaller values of $\Theta$ (see the different slopes in the same figures). This is especially true for the symmetry broken state and we attribute this property to the vicinity of the Gaussian fixed point.

Table 2: Values of the discontinuities.

\begin{tabular}{||c|c|c|c|c||}
\hline$\Theta$ & $s_{m f}$ & $\Delta\left\langle O_{3}\right\rangle$ & $\frac{\Delta\left\langle O_{1}\right\rangle}{\Delta\left\langle O_{3}\right\rangle^{2}}$ & $\frac{\Delta\left\langle O_{2}\right\rangle}{\Delta\left\langle O_{3}\right\rangle^{2}}$ \\
\hline 1 & 1.312 & - & - & - \\
\hline 3 & 2.285 & $1.603(50)$ & $0.955(70)$ & $0.946(69)$ \\
\hline 4 & 2.639 & $2.036(05)$ & $0.998(07)$ & $0.999(07)$ \\
\hline 5 & 2.951 & $2.458(05)$ & $0.991(06)$ & $1.000(07)$ \\
\hline
\end{tabular}

\subsection{The Order Parameter Jump}

In Fig 15) we show as a function of $\Theta$ the order parameter jump $\Delta\left\langle O_{3}\right\rangle$ scaled by the mean field result. The actual magnitude of the order parameter jump comes out smaller, than predicted by the mean field analysis. In addition we also observe a mild deviation from scaling and it appears as if possible scaling deviations might be parametrized by a correction linear in $\Theta$. Fig. 15) contains a corresponding fit (dotted line in the figure), which predicts that the order parameter jump in units of the mean field order parameter discontinuity might in the continuum limit $\Theta \rightarrow 0$ be as low as one half. However, the theoretical status of scaling corrections in 3-dimensions is unclear [18] and in our context we might regard such an extrapolation as a lower bound to the 
true value of the order parameter jump. Further simulations closer to the continuum are therefore required. They may show a bending of the order parameter discontinuity to somewhat larger values than half of the mean field result, but will be significantly lower than the mean field prediction. Such a result is consistent with the variational upper bound nature of the mean field approximation. Employing our extrapolation we currently estimate the order parameter discontinuity to be

$$
\frac{\Phi\left(T_{c}\right)}{T_{c}}=0.68(4)
$$

Using the $T_{c}$ determination we obtain $\Phi\left(T_{c}\right)=78(5) \mathrm{GeV}$ at a physical Higgs mass value of $m_{H}=37.16 \mathrm{GeV}$.

\subsection{The Latent Heat}

The volume normalized latent heat in units of $T_{c}^{4}, \frac{\mathcal{L}}{T_{c}^{4}}$, is obtained from the expectation value of the internal energy $\frac{\mathcal{U}}{T^{4}}$

$$
\left\langle\frac{\mathcal{U}}{T^{4}}\right\rangle=\left\langle\frac{1}{\Theta^{3}} \beta \partial_{\beta} S(\beta)\right\rangle
$$

by calculating its difference in the symmetric and the symmetry broken state at $T_{c}$.

$$
\frac{\mathcal{L}}{T_{c}^{4}}=\left.\left\langle\frac{\mathcal{U}}{T_{c}^{4}}\right\rangle\right|_{S}-\left.\left\langle\frac{\mathcal{U}}{T_{c}^{4}}\right\rangle\right|_{S B}
$$

Hereby $\beta$ denotes the inverse temperature $\beta=\frac{1}{T}$ and $S$ denotes the action of the $3+1$ dimensional theory. We note that our convention in defining the latent heat is opposite to what we have used for the order parameter discontinuities. This makes the latent heat positive. Incorporating the full temperature dependence of all of our physical couplings we obtain for the internal energy in terms of the 3-dimensional reduced theory the expression

$$
\begin{gathered}
\left\langle\frac{\mathcal{U}}{T^{4}}\right\rangle=\left\langle\frac{S_{3 D, l a t}}{\Theta^{3}}+\sum_{x}\left\{-\left(\frac{3 g^{2}}{8}+\frac{\lambda}{6}-\frac{m_{H}^{2}}{16 \pi^{2} T^{2}}\left(\frac{9}{4} g^{2}+\lambda\right) \frac{\psi_{x}^{+} \psi_{x}}{\Theta}\right.\right.\right. \\
-\frac{1}{32 \pi^{2}}\left(\frac{9 g^{4}}{16}+\frac{\lambda^{2}}{3}+\frac{3 \lambda g^{2}}{4}\right)\left(\frac{\psi_{x}^{+} \psi_{x}}{\Theta}\right)^{2} \\
+\frac{g^{3}}{16 \pi}\left[\left(\left(\frac{2 \gamma_{B} g}{3 \pi}\right)^{2}+\frac{\psi_{x}^{+} \psi_{x}}{\Theta}\right)^{\frac{3}{2}}+\frac{1}{2}\left(\frac{10}{3}+\frac{\psi_{x}^{+} \psi_{x}}{\Theta}\right)^{\frac{3}{2}}\right] \\
\left.\left.+\frac{3 g^{3}}{8 \pi}\left[\left(\frac{\gamma_{B} g}{3 \pi}\right)^{2}\left(\left(\frac{2 \gamma_{B} g}{3 \pi}\right)^{2}+\frac{\psi_{x}^{+} \psi_{x}}{\Theta}\right)^{\frac{1}{2}}+\frac{5}{12}\left(\frac{10}{3}+\frac{\psi_{x}^{+} \psi_{x}}{\Theta}\right)^{\frac{1}{2}}\right]\right\}\right\rangle
\end{gathered}
$$


We note here that the latent heat calculation requires the calculation of discontinuities of more complicated operators e.g., the calculation of discontinuities in $\sum_{x}\left(\psi_{x}^{+} \psi_{x}\right)^{2}$ and discontinuities in certain functions of the fields. Since we did not measure all required operators directly, we assume in the numerical analysis that corresponding discontinuities show a mean field like behavior e.g., the discontinuity in the operator $\sum_{x}\left(\psi_{x}^{+} \psi_{x}\right)^{2}$, for instance, is replaced by the discontinuity in the operator $O_{3}$ to the fourth power. Our data support such an assumption, at least, for the restricted set of operators at the large values of $\Theta$, considered in the present investigation, see Table 2) and the related discussion.

In Fig. 16) we display the discontinuity of the volume normalized 3dimensional action eq.(28) scaled by corresponding $\Theta$-powers at the critical point (circles) as a function of $\Theta$. It is consistent with being zero and results from a cancellation of positive and negative terms, which can be attributed to the kinetic term and the potential term in the action. As the action gap itself is related to the pressure, which is continous even at the first order phase transition, this is an expected behavior and merely is a check on the consistency of our analysis.

The latent heat per unit volume $\frac{\mathcal{L}}{T_{c}^{4}}$ also appears in Fig. 16). The triangles denote the results of our Monte Carlo simulation, while the crosses (constant with $\Theta$ ) correspond to the mean field analysis of the theory. Again we observe a significant weakening of the phase transition as compared to the mean field treatment. We also observe scaling deviations i.e., as a function of $\Theta$ the latent heat values have not yet settled to their continuum value. Here we quote the value of the latent heat obtained from simulations at $\Theta=3$ :

$$
\frac{\mathcal{L}}{T_{c}^{4}}=0.122(8) \text {. }
$$

This value again is less than half of the mean field result $\frac{\mathcal{L}}{T_{c}^{4}}=0.262$ and should be close to the continuum result provided further scaling deviations turn out not to be too large.

\subsection{The Interface Tension}

Following Binder [19] the interface tension inbetween symmetric and symmetry broken states of the theory can be obtained from the shape of the 
probability distribution functions at $\kappa_{e h}$. Denoting the value of the maxima of the probability distribution functions $P^{\max }\left(O_{i}\right)$, states inbetween the two maxima correspond to mixed phases, which on the largest lattices form an interface with a cross sectional area $L^{2}$. Because of the periodic boundary conditions there are actually two interfaces in the system and the total interfacial area thus is $2 L^{2}$. Denoting the value of the probability distribution function at its minimum $P^{\min }\left(O_{i}\right)$, the interface tension can be defined by the limit

$$
\sigma_{\text {lat }}=\lim _{L \rightarrow \infty} \frac{1}{2 L^{2}} \ln \left[\frac{P^{\max }\left(O_{i}\right)}{P^{\min }\left(O_{i}\right)}\right] \quad i=1,2,3 .
$$

The quantity $\sigma_{\text {lat }}$ is connected to the continuum interface tension $\sigma$ via $\sigma_{\text {lat }}=\frac{\sigma}{T_{c}^{3}} \Theta^{2}$. It is however expected that the extrapolation to infinite volume is more subtle, than in the case of discontinuities in the order parameter. Interfaces in finite boxes show sizable fluctuations controlled by their stiffness and finite volume corrections to $\sigma_{\text {lat }}$ may have a complicated $L$-dependent analytical form, as is demonstrated by numerical simulations in spin models [20]. In Fig. 17) and Fig. 18) we exhibit our interface tension analysis. Fig. 17) displays the quantity $\ln \left(\frac{P^{\max }\left(O_{1}\right)}{P^{\min }\left(O_{1}\right)}\right)$ for three $\Theta$-values as a function of the expected interfacial cross section $L^{2}$ for all considered lattice sizes. In particular for $\Theta=5$ we observe a very fast rise with $L^{2}$, indicating that mixed phase configurations are suppressed by many order of magnitudes in the path integral and that the interface tension thus is large. The data are also roughly consistent with a linear increase of the considered quantity with the interfacial cross section, though some curvature to larger slopes is indicated. Since the status of possible finite volume correction terms to our measured quantities is unclear, we may regard the straight line fits of Fig. 17) as estimates for the expected surface tensions values. They are displayed in Fig. 18) as functions of $\Theta$ in units of the corresponding mean field result obtained in the thin wall approximation, eq.(40). They show indeed a dramatic difference. In the mean field calculation we expect at the given couplings a behavior according to $\sigma_{\text {lat }}=0.0242 \Theta^{2}$. Definitely large scaling deviations are observed, while at the same time e.g., at $\Theta=3$, our estimates are about 37 times smaller than the corresponding mean field calculation result. Thus it is indicated here, that the interface tension is subject to large corrections due to the fluctuations of the scalar field. Turning our interface calculation into physical numbers the data point at $\Theta=3$ predicts an interface tension 
of as low as about $960 \mathrm{GeV}^{3}$, which in the current context may be viewed as an order of magnitude estimate. The numerical situation is not totally satisfying and in the future one should also explore other possibilities for interface tension determinations, like simulations on asymmetric lattices and studies of tunneling masses on such geometries.

\section{Conclusion}

In this paper a pure scalar model has been proposed for the description of the finite temperature electroweak phase transition (EWPT). This effective model was obtained from the full $3+1$ dimensional field theory through integration over the non-static Matsubara modes, realised in the 1-loop approximation. The remaining 3-dimensional gauge degrees of freedom and the effective adjoint Higgs-field were eliminated in a subsequent Gaussian integration step.

Intuitive justification for such scenario is provided by the thermal mass hierarchy observed in the perturbative treatment of EWPT for moderately small Higgs masses. Also, we have demonstrated that in an appropriately defined large $\mathrm{N}$ limit the second integration step is exact.

The resulting model is a 3-dimensional $\mathrm{O}(4)$-invariant ferromagnet with cubic and quartic potential.

Its discretisation and the proper procedure for taking the continuum limit of its phase transition have been thoroughly explained. Especially, the sensitivity of the $T_{c}$ dependence on $m_{H}(T=0)$ stemming from the application of different renormalisation conditions in the continuum has been discussed in detail.

The mean field solution of the lattice system and its continuum limit has reproduced in all aspects the results of the improved 1-loop perturbative treatments of EWPT $[2,3]$ (influence of magnetic screening, order parameter discontinuity, surface tension, etc.). Also it called attention to the dramatic sensitivity of $T_{c}$ to the renormalisation conditions for $m_{H}(T=0)<30 \mathrm{GeV}$. This circumstance has prevented the discussion of the possible existence of a lower limiting Higgs mass value below which the gauge symmetry could not be restored at any finite temperature. 
The mean field analysis served for orienting the numerical calculations, performed at $m_{H}(T=0) \sim 35 \mathrm{GeV}$. This value has been selected : i) to have a strong signal of a first order transition, ii) to be free of the uncertainties introduced by the renormalisation prescriptions, iii) to be able to compare the results of a careful numerical study with other computer studies of EWPT based on different effective 3-d models. 88

The numerical data consistently exhibit at the considered value of the Higgs mass a weaker first order phase transition as compared to the zeroth order mean field approximation of the theory. Discontinuities of the theory like the order parameter jump and the latent heat turn out to be somewhat smaller than corresponding mean field results. The critical temperature $T_{c}=$ $114 \mathrm{GeV}$ appears larger than mean field, again consistent with a weakening of the first order phase transition. The interface tension is strongly affected by the fluctuations of the scalar field and its value will be much lower than corresponding mean field predictions. By themselves these findings may not be regarded as surprises as naively one expects the fluctuations of the Higgs field to work in the observed direction. Similar experience has been gained from numerical estimations of the interface tension of the SU(3) pure gluon theory.

Comparing our results with the results of another $\mathrm{MC}$ simulation making use of another variant of 3-dimensional effective models of the electroweak phase transition at comparable value of the Higgs mass, we make, however, an interesting observation. While the authors of [8] keep the gauge fields in the 3-dimensional Gauge-Higgs model as dynamical degrees of freedom in their simulation, their findings indicate a strengthening of the first order phase transition, when compared to a one-loop perturbative analysis of their model, and also, when compared to the results of our simulation. They do quote e.g., a lower value of $T_{c}=85 \mathrm{GeV}$ at a value of $m_{H}=35 \mathrm{GeV}$ or a value of the order parameter discontinuity of about $170 \mathrm{GeV}$, while our value $\Phi\left(T_{c}\right)=78(5) \mathrm{GeV}$ is about half as large. Thus it appears as if the non-perturbative dynamics of gauge fields in the dimensionally reduced model works in a direction opposite to the effect of the fluctuations of the Higgs field, and eventually dominates the physical characteristics of the phase transition.

Acknowledgments: We thank the computer centers at HLRZ, RWTHAachen and at the University of Cologne for providing us with computer time 
on their vector machines. We are indebted to $\mathrm{W}$. Buchmüller for a useful discussion. We also acknowledge the support of the EEC through contract numbers ERB-CHRX-CT-92-0051 and ERB-CIPA-CT-92-2061. 


\section{References}

[1] M. Dine, R. G. Leigh, P. Huet, A. Linde and D. Linde, Phys. Rev D46 (1992) 550 .

[2] W. Buchmüller, Z. Fodor, T. Helbig and D. Walliser, The weak electroweak phase transition, DESY 93-021, February 1993. (to appear in Ann. of Phys.)

[3] J. R. Espinosa, M. Quiros and F. Zwirner,Phys. Lett. B314 (1993) 206.

[4] M. Gleiser and E. W. Kolb, Phys. Rev. D48 (1993)1560.

[5] M. Alford and J. March-Russel, Nucl. Phys. B417 [FS] (1994) 527.

[6] P.Arnold ans L.G. Yaffe, Phys. Rev. D49 (1994) 3003

[7] B. Bunk, E.M. Ilgenfritz, J. Kripfganz and A. Schiller, Phys. Lett. B284 (1992) 371 and Nucl. Phys. B403 (1993) 453.

[8] K. Kajantie, K. Rummukainen and M. Shaposhnikov, Nucl. Phys. B407 (1993) 356;

[9] K. Farakos, K. Kajantie, K. Rummukainen and M. Shaposhnikov, 3D Physics and the Strength of the Electroweak Phase Transition: Perturbation Theory, CERN-TH. 6973/94.

[10] D. Bödeker, W. Buchmüller, Z. Fodor and T. Helbig, Aspects of the Cosmological Electroweak Phase Transition, DESY 93-147, Nov. 1993.

[11] V. Jain and A. Papadopoulos, Phys. Lett. 303 (1993) 315.

[12] A. Jakovác, K. Kajantie and A. Patkós, A Hierarchy of Effective Field Theories of Hot Electroweak Matter, TFT-HU-94-01, 1994 Jan. (to appear in Phys. Rev. D)

[13] A.D. Linde, Reports on Progs. Phys. 42 (1979) 389.

[14] T. Banks and A. Zaks, Nucl. Phys. B196 (1982) 189.

[15] P. Salomonson and B.S. Skagerstam, Phys. Lett. 155B (1985) 100. 
[16] B.A. Berg and T. Neuhaus, Phys. Lett. B267 (1991) 249.

[17] J. Lee and J.M. Kosterlitz, Phys. Rev. B43 (1991) 3265; C. Borgs and R. Kotecký, J. Stat. Phys. 61 (1990) 79; C. Borgs, R. Kotecký and S. Miracle-Sole, J. Stat. Phys. 62 (1991) 529.

[18] J. Zinn-Justin, Quantum Field Theory and Critical Phenomena, Oxford Science Publications.

[19] K. Binder, Z. Phys. B43 (1981) 119; K. Binder, Phys. Rev. A25 (1982) 1699.

[20] A. Billoire, T. Neuhaus and B.A. Berg, Nucl. Phys. B413 (1994) 795, B.A. Berg, U. Hansmann and T. Neuhaus, Z.Phys. B90 (1993) 229. 
This figure "fig1-1.png" is available in "png" format from: http://arxiv.org/ps/hep-lat/9406012v1 
This figure "fig1-2.png" is available in "png" format from: http://arxiv.org/ps/hep-lat/9406012v1 
This figure "fig1-3.png" is available in "png" format from: http://arxiv.org/ps/hep-lat/9406012v1 
This figure "fig1-4.png" is available in "png" format from: http://arxiv.org/ps/hep-lat/9406012v1 
This figure "fig1-5.png" is available in "png" format from: http://arxiv.org/ps/hep-lat/9406012v1 
This figure "fig1-6.png" is available in "png" format from: http://arxiv.org/ps/hep-lat/9406012v1 
This figure "fig1-7.png" is available in "png" format from: http://arxiv.org/ps/hep-lat/9406012v1 
This figure "fig1-8.png" is available in "png" format from: http://arxiv.org/ps/hep-lat/9406012v1 
This figure "fig1-9.png" is available in "png" format from: http://arxiv.org/ps/hep-lat/9406012v1 
This figure "fig1-10.png" is available in "png" format from: http://arxiv.org/ps/hep-lat/9406012v1 
This figure "fig1-11.png" is available in "png" format from: http://arxiv.org/ps/hep-lat/9406012v1 
This figure "fig1-12.png" is available in "png" format from: http://arxiv.org/ps/hep-lat/9406012v1 
This figure "fig1-13.png" is available in "png" format from: http://arxiv.org/ps/hep-lat/9406012v1 
This figure "fig1-14.png" is available in "png" format from: http://arxiv.org/ps/hep-lat/9406012v1 
This figure "fig1-15.png" is available in "png" format from: http://arxiv.org/ps/hep-lat/9406012v1 
This figure "fig1-16.png" is available in "png" format from: http://arxiv.org/ps/hep-lat/9406012v1 
This figure "fig1-17.png" is available in "png" format from: http://arxiv.org/ps/hep-lat/9406012v1 
This figure "fig1-18.png" is available in "png" format from: http://arxiv.org/ps/hep-lat/9406012v1 